University of Warwick institutional repository: http://go.warwick.ac.uk/wrap This paper is made available online in accordance with publisher policies. Please scroll down to view the document itself. Please refer to the repository record for this item and our policy information available from the repository home page for further information.

To see the final version of this paper please visit the publisher's website. Access to the published version may require a subscription.

Author(s): A. D. Capewell, T. J. Grasby, T. E. Whall, and E. H. C. Parker Article Title: Terrace grading of SiGe for high-quality virtual substrates Year of publication: 2002

Link to published version: http://dx.doi.org/10.1063/1.1529308

Publisher statement: None 


\title{
Terrace grading of SiGe for high-quality virtual substrates
}

\author{
A. D. Capewell, ${ }^{\text {a) }}$ T. J. Grasby, T. E. Whall, and E. H. C. Parker \\ Department of Physics, University of Warwick, Coventry CV4 7AL, United Kingdom
}

(Received 13 August 2002; accepted 22 October 2002)

\begin{abstract}
Silicon germanium $(\mathrm{SiGe})$ virtual substrates of final germanium composition $x=0.50$ have been fabricated using solid-source molecular beam epitaxy with a thickness of $2 \mu \mathrm{m}$. A layer structure that helps limit the size of dislocation pileups associated with the modified Frank-Read dislocation multiplication mechanism has been studied. It is shown that this structure can produce lower threading dislocation densities than conventional linearly graded virtual substrates. Cross-sectional transmission electron microscopy shows the superior quality of the dislocation network in the graded regions with a lower rms roughness shown by atomic force microscopy. X-ray diffractometry shows these layers to be highly relaxed. This method of Ge grading suggests that high-quality virtual substrates can be grown considerably thinner than with conventional grading methods.
\end{abstract}

(C) 2002 American Institute of Physics. [DOI: 10.1063/1.1529308]

As fundamental limits are reached in device size, silicon germanium's ( $\mathrm{SiGe}$ ) promise of greater device speed without the need for device scaling has become increasingly attractive. If the inherent strain in this lattice-mismatched system can be properly managed, carrier mobilities significantly higher than that of bulk silicon can be achieved. To tailor the properties of the SiGe system the strain in the active layers must be controlled. This is achieved by growing a relaxed SiGe buffer layer termed a "virtual substrate." In addition, the tunable lattice constant of SiGe virtual substrates offers the possibility of incorporating III-V semiconductors into silicon complementary metal-oxide semiconductors for optoelectronics applications.

In order to relax the virtual substrate, a layer in excess of the critical thickness must be grown. ${ }^{1}$ If this layer is of uniform $\mathrm{Ge}$ composition, the excessive biaxial strain is relieved by the formation of misfit dislocations at the $\mathrm{Si} / \mathrm{SiGe}$ interface. In the absence of pre-existing dislocations, dislocation half-loops are generated at the surface and expand to the $\mathrm{Si} / \mathrm{SiGe}$ interface. The misfit dislocations extend by the gliding of threading dislocations that connect each end of the dislocation to the surface. Threading dislocations can seriously degrade the quality of any device fabricated on a virtual substrate, so their density must be kept low. ${ }^{2}$ Since the network of misfit dislocations is confined to a single atomic plane (the interface between the $\mathrm{Si}$ and $\mathrm{SiGe}$ ) the dislocations interact and can become blocked. ${ }^{2,3}$ Further relaxation must occur by nucleation of more dislocations. This leads to a large density of threading dislocations, which is detrimental to the quality of the virtual substrate. More commonly, the germanium composition is graded over the buffer layer so that the misfit dislocations form on many different planes, not just on the growth interface. Consequently, the interactions between dislocations are greatly reduced. This in turn reduces the density of threading dislocations by several orders of magnitude.

One problem with the linearly graded virtual substrate is that the mechanism most commonly responsible for relax-

${ }^{a)}$ Electronic mail: a.d.capewell@warwick.ac.uk ation, the modified Frank-Read (MFR) mechanism, ${ }^{4}$ generates many dislocations from a few well-spaced sources. This causes dislocations to be piled up on the same (111) atomic glide planes. Since each dislocation causes a small surface step on the wafer, ${ }^{5}$ the dislocation pileups disrupt the surface with large amplitude undulations known as crosshatch. This crosshatch causes problems with device processing and can lead to pinning of the threading dislocations at the surface. ${ }^{2}$

In this letter, a "terrace-grading" system is studied to reduce the size of these pileups. The germanium composition is graded linearly to $x=0.10$, and a uniform layer is then grown to ensure the dislocations generated in the graded region glide as far as possible. The dislocations are then confined to the graded layer. This growth pattern is then repeated in steps of $x=0.10$ up to the final composition, each step consisting of a linearly graded layer containing the misfit dislocations, and a uniform layer separating the pileups. In this technique, each layer of the virtual substrate is allowed to relax by the MFR mechanism, but the dislocation pileups should be contained within each of the graded layers and should be independent from each other. Consequently, the amplitude of the associated crosshatch caused by the pileups is reduced, since the pileups are now smaller and more evenly distributed.

All samples were grown on 4 in. Si (001) substrates using a VG Semicon V90S solid source molecular beam epitaxy system. The wafers were first cleaned in a modified RCA etch followed by a $2 \%$ HF dip and an in-situ desorb at $890^{\circ} \mathrm{C}$ for $20 \mathrm{~min}$. The temperature was reduced while growing $100 \mathrm{~nm}$ of $\mathrm{Si}$, so that the growth of the virtual substrate could commence without interruption.

Initially, two virtual substrates were grown with final germanium composition of $x=0.50$. The first wafer, designated TG1, was grown with a terrace graded germanium profile, as described earlier. The uniform and graded layers were all $200 \mathrm{~nm}$ thick, making the total thickness of the virtual substrate $2 \mu \mathrm{m}$. The growth temperature was reduced with increasing germanium composition in order to suppress surface roughening. ${ }^{6}$ The growth temperature was maintained at $700{ }^{\circ} \mathrm{C}$ during the first graded layer and $x=0.10$ uniform 

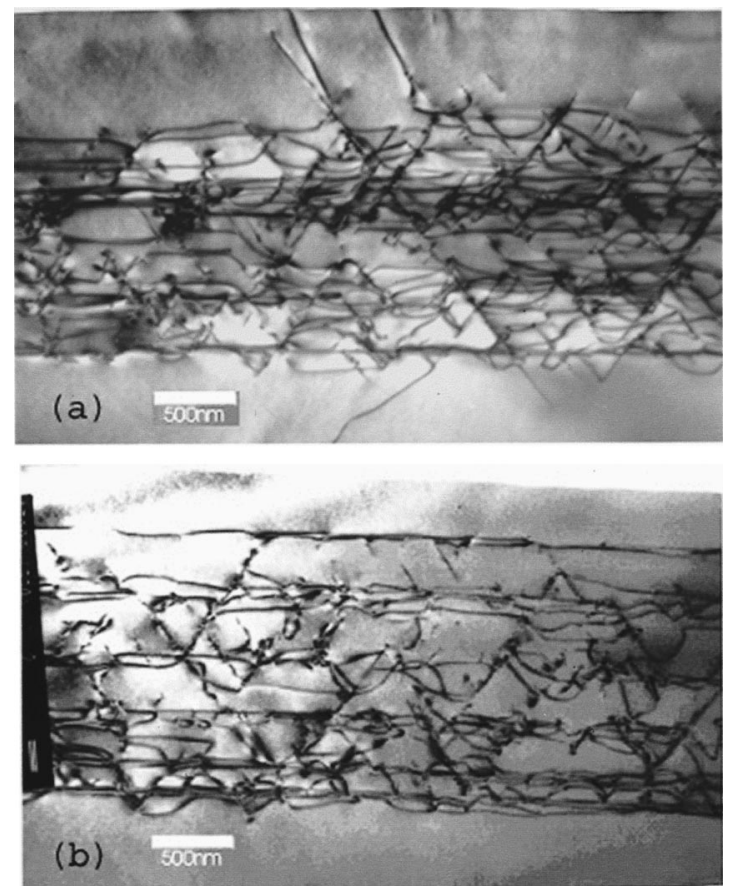

FIG. 1. (a) XTEM micrograph of LG1 showing large dislocation pileups and threading dislocations. (b) XTEM micrographs of TG1 showing that dislocation pileups are contained within the graded region and no threading dislocations penetrate the final uniform layer (both scale bars are $500 \mathrm{~nm}$ ).

layer. The $x=0.20,0.30,0.40$, and 0.50 uniform layers were grown at $650,600,575$, and $550{ }^{\circ} \mathrm{C}$, respectively. The temperature was linearly ramped between these values during the growth of the graded layers. The second wafer, designated LG1, was grown with a conventional linear grade from $x=0.0$ to 0.50 over $2 \mu \mathrm{m}$, with the temperature linearly ramped from 700 to $550^{\circ} \mathrm{C}$ during the growth.

The virtual substrates were examined by cross-sectional transmission electron microscopy (XTEM) in a JEOL JEM2000FX TEM using an accelerating voltage of $200 \mathrm{kV}$. The samples were mechanically thinned and ion milled to perforation. All micrographs were taken in the bright field using the two-beam condition with diffraction vector $\mathbf{g}$ set to (220) for high dislocation contrast. The surface morphology of the virtual substrates was examined using a Digital Instruments Nanoscope III atomic force microscope (AFM) in contact mode. X-ray diffractometry (XRD) was carried out in order to confirm the germanium composition and state of relaxation. $^{7}$

The XTEM micrographs of the samples LG1 and TG1 are shown in Fig. 1. The micrograph of the linearly grade virtual substrate (LG1) shown in Fig. 1(a) shows that the pileups associated with the MFR mechanism extend through the entire virtual substrate. In addition, some dislocations have penetrated into the uniform capping layer to the surface. In contrast, the dislocation network of TG1, shown in Fig. 1(b), shows that the dislocations are mostly confined to the graded regions of the virtual substrate. Dislocation pileups characteristic of the MFR relaxation mechanism can be clearly seen, but these do not extend through the entire virtual substrate. Instead, the pileups only penetrate through one, or sometimes two, of the graded sections. It is evident

FIG. 2. SIMS profiles of samples TG1 and LG1.
that the uniform layers separate the MFR pileups from each
Downloaded 06 Jul 2009 to 137.205.202.8. Redistribution subject to AIP license or copyright; see http://apl.aip.org/apl/copyright.jsp
TABLE I. Summary of germanium composition and relaxation of the uniform layers of sample TG1 measured by XRD.

\begin{tabular}{lccccc}
\hline \hline & Layer 1 & Layer 2 & Layer 3 & Layer 4 & Layer 5 \\
\hline Composition, $x$ & 0.094 & 0.184 & 0.289 & 0.397 & 0.542 \\
Relaxation & $101 \%$ & $102 \%$ & $101 \%$ & $100 \%$ & $95 \%$ \\
\hline \hline
\end{tabular}

of the graded sections, with no evidence of any dislocations penetrating the final uniform capping layer.

XRD was carried out on sample TG1 using symmetric (004) and asymmetric (224) scans. Table I summarizes the results. As can be seen, the intended compositions of $x$ $=0.10,0.20,0.30,0.40$, and 0.50 for the uniform layers have been closely achieved, with each layer exhibiting a high state of relaxation. The lower levels are over $100 \%$ relaxed, which is an indication that they have become tensile strained. This may be due to an oversaturation of misfit dislocations which will over-relax the layers. ${ }^{8}$ This suggests that the MFR mechanism has pushed dislocations from the higher layers into the relaxed lower layers, causing higher dislocation densities than is required for complete relaxation. This is supported by Fig. 1(b), in which higher dislocation densities can be seen in the lower layers.

Secondary ion mass spectrometry (SIMS) was carried out to check the grading profiles of samples LG1 and TG1. Figure 2 shows the SIMS data for the two samples using normal incidence $\mathrm{O}_{2}^{+}$ions at $1 \mathrm{keV}$. The SIMS data confirms that the desired terrace grading profile has been achieved, although the uniform layers in the lower levels seem to have become slightly graded. It is believed that this is an artifact of the SIMS analysis due to uneven erosion rates through such deep, highly dislocated structures. The uniformity of the lower levels has been confirmed by energy dispersive spectroscopy (EDS), and shall be reported elsewhere. The SIMS shows that the linearly graded sample (LG1) is of lower final composition than the terrace graded sample, and this has also been confirmed by EDS. Since the quality of the virtual substrates is expected to decrease with higher grading rate, this only exaggerates the superiority of the terrace graded profile.

AFM measurements have been taken to examine the surface topography of the two samples. Figure 3 shows typical line profiles taken from a $10 \mu \mathrm{m} \times 10 \mu \mathrm{m}$ scan of both samples. It can be seen that LG1 is characterized by regularly spaced peaks and troughs, with a period of approxi-

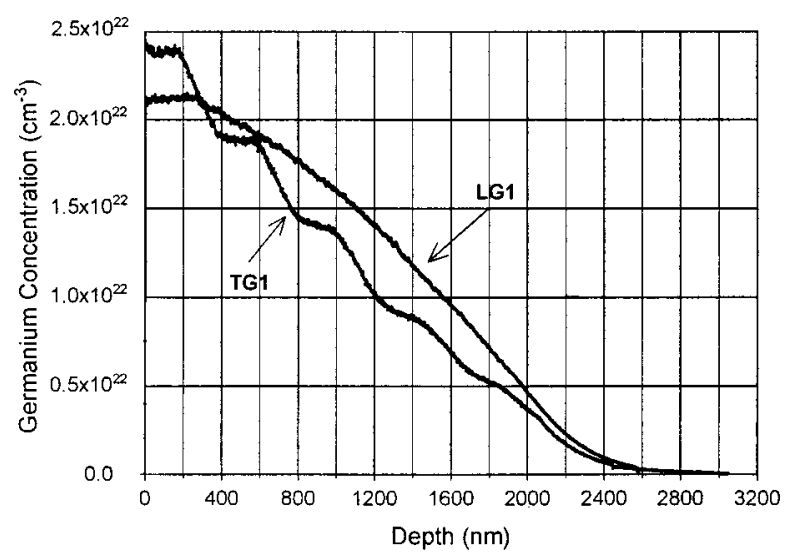

FIG. 2. SIMS profiles of samples TG1 and LG1. of copyright; see http://apl.aip.org/apl/copyright.jsp 


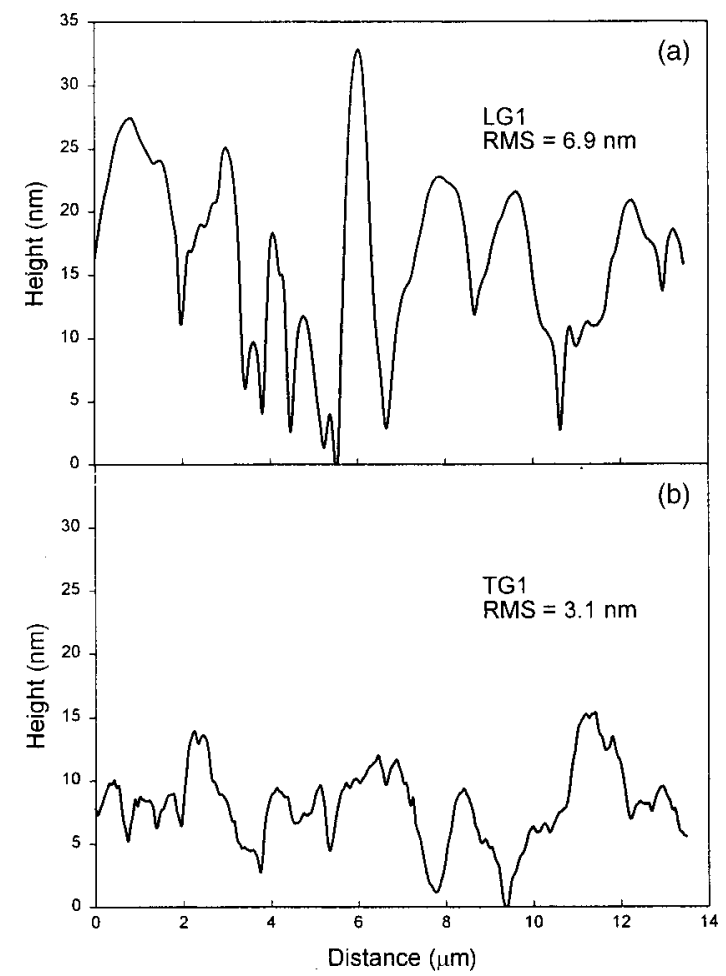

FIG. 3. AFM profiles of (a) sample LG1 and (b) sample TG1 plotted to the same scale.

mately $1 \mu \mathrm{m}$. This is characteristic of the crosshatch pattern common in layers relaxed by the MFR mechanism. In contrast, the profile of sample TG1 shows more closely spaced peaks, with a much lower amplitude than for LG1. This is in agreement with the hypothesis that the pileups associated with the MFR mechanism have been reduced in magnitude, and are more evenly distributed across the wafer. The rms roughness of sample TG1 is $3.1 \mathrm{~nm}$, compared with an rms roughness for LG1 of $6.9 \mathrm{~nm}$. Threading dislocation densities were determined by Nomarski microscopy after a Schimmel etch, ${ }^{9}$ and found to be $1.0 \times 10^{6} \mathrm{~cm}^{-2}$ and 7.0 $\times 10^{6} \mathrm{~cm}^{-2}$ for samples TG1 and LG1, respectively. This shows clearly that the terrace graded approach is superior to the conventional linear grading technique. Although higher quality virtual substrates have been reported, usually by lin- ear grading using other growth techniques, this grading profile could be incorporated into these techniques to give further improvements.

Electrically active Ge channels have been grown on virtual substrates using this terrace grading technique. Early results have shown very high-quality channels and promising electrical results, with a hole mobility of $14900 \mathrm{~cm}^{2} \mathrm{~V}^{-1} \mathrm{~s}^{-1}$ at $10 \mathrm{~K}$ and a sheet density of $8.5 \times 10^{11} \mathrm{~cm}^{-2}$. Further work is expected to show considerable improvements, and is to be reported elsewhere.

In conclusion, we have shown that the terrace grading profile for producing SiGe virtual substrates is superior to a conventional linearly graded profile under identical growth conditions. XRD has shown that the virtual substrate is highly relaxed, and XTEM has shown that the dislocation pileup associated with the MFR mechanism has been controlled. AFM has shown a much smoother surface than for the linear graded wafer. This growth profile shows clear advantages over conventional linear grading and should, for a given terminating value of $x$, allow the thickness of the virtual substrate to be considerably reduced. Additionally, this technique can be extended to any Ge composition, and should be achievable by any standard epitaxial growth technique.

The authors are grateful to R. J. H. Morris of the University of Warwick for the SIMS profiles and J. Zhang of Imperial College, London for the XRD.

${ }^{1}$ J. W. Matthews and A. E. Blakeslee, J. Cryst. Growth 27, 118 (1974).

${ }^{2}$ E. A. Fitzgerald and S. B. Samavedam, Thin Solid Films 294, 3 (1997).

${ }^{3}$ E. A. Stach, K. W. Schwarz, R. Hull, F. M. Ross, and R. M. Tromp, Phys. Rev. Lett. 84, 947 (2000).

${ }^{4}$ F. K. Legoues, B. S. Meyerson, J. F. Morar, and P. D. Kirchner, J. Appl. Phys. 71, 4230 (1992).

${ }^{5}$ M. A. Lutz, R. M. Feenstra, F. K. LeGoues, P. M. Mooney, and J. O. Chu, Appl. Phys. Lett. 66, 724 (1995).

${ }^{6}$ P. M. Mooney, J. L. Jordan-Sweet, J. O. Chu, and F. K. LeGoues, Appl. Phys. Lett. 66, 3642 (1995).

${ }^{7}$ J. M. Hartmann, B. Gallas, R. Ferguson, J. Fernadez, J. Zhang, and J. J. Harris, Semicond. Sci. Technol. 15, 362 (2000).

${ }^{8}$ J. Michel, E. A. Fitzgerald, Y.-H. Xie P. S. Silverman, M. Morse, and L. C. Kimerling, J. Electron. Mater. 21, 1099 (1992).

${ }^{9}$ D. G. Schimmel, J. Electrochem. Soc. 123, 734 (1976). 